\title{
Deep Learning for Financial Time-Series Data Analytics: An Image Processing Based Approach
}

\author{
Watthana Pongsena, Prakaidoy Ditsayabut, Nittaya Kerdprasop, and Kittisak Kerdprasop
}

\begin{abstract}
Forex or Foreign Exchange is the largest financial market with a huge amount of daily trading volume. Traditionally, the tool or strategy that Forex traders usually used is divided into Fundamental Analysis and Technical Analysis. Presently, the world computational technological advance, such as Artificial Intelligence plays a significant role in the financial domain. The various existing research applies Machine Learning and Deep Learning to develop powerful models that can be used as a tool for traders in order to develop their trading tools or strategies. However, most existing models are developed targeting for the stock market. There are still lag of research that applies the modern Machine Learning or Deep Learning for predicting the movement of the price in the Forex market. In this paper, we propose a novel predicting model based on Deep Convolutional Neural Network that can be effectively used as a tool in order to make the profits for Forex traders. We evaluate the performance of the proposed CNN model from two perspectives. The first perspective is to evaluate the accuracy of the prediction and the second perspective is to evaluate the ability to make profits. The experimental results show that in term of accuracy of the prediction, our proposed CNN model provide the accuracy up to approximately $77 \%$. This result is similar to the C5.0 algorithm, which is a rule-based algorithm of Machine Learning. The results are different in a trivial faction. In terms of financial perspective, the proposed CNN model performs well as it produces approximately $\$ 69 \mathrm{~K}$ for one and a half year (from January 2017 to September 2018).
\end{abstract}

Index Terms-Machine learning, deep learning, long short-term memory, simulated annealing, time-series data analytic, foreign exchange market.

\section{INTRODUCTION}

Foreign Exchange (Forex or FX) is the biggest financial market in the world [1] where the pairwise of many currencies around the world are traded. Like others financial markets, traders who invest in forex market require a good tool or trading strategy in order to make their profits. Typically, there are two types of tool or strategy that Forex trader can be exploited including Fundamental Analysis (FA) [2] and Technical Analysis (TA) [3]. FA is a method that attempts to measure the actual value of the security by examining its values related to the current economic, financial reports, other qualitative and quantitative factors. On the other hands, TA uses the information of in chart value

Manuscript received April 3, 2019; revised November 6, 2019.

Watthana Pongsena, Nittaya Kerdprasop, and Kittisak Kerdprasop are with the School of Computer Engineering, Suranaree University of Technology, Nakhon Ratchasima 30000 Thailand (e-mail: pongsena@hotmail.com,nittaya.k@sut.ac.th,kerdpras@sut.ac.th).

Prakaidoy Ditsayabut is with the School of Biotechnology, Suranaree University of Technology, Nakhon Ratchasima 30000 Thailand (e-mail: prakaidoy_sut@hotmail.com). according to the time-series historical data to create the powerful measurement tool for evaluating the value of the stock or the quotation of a currency pairwise. In the Forex market, TA is much more popular than FA because TA is easier to used [4], and it can eliminate the complexity to analyze the financial terms [5]. TA believes that there is unnecessary to analyze the financial or economic data since all the relevant information already includes in the chart. Therefore, a trader, who employ TA allows to focus on analyzing the chart itself for hints into where the quotation may be heading. Presently, there are plenty of tools also known as technical indicators that we can use for TA, such as, Moving Average Convergence Divergence (MACD) [6], Relative Strength Index (RSI) [7], Stochastic oscillator [8], etc. Although analyzing the chart using technical indicators is quick and easy, traders often questioned that which indicators could be used and what are the most suitable parameters could be set for each indicator.

In the last decade Artificial Intelligence (AI), the world computational technological advance, is widely applied in the financial domain. Various Machine Learning algorithms, such as Artificial Neural Network (ANN) [9]-[12] and an optimization algorithm, such as genetic algorithm (GA) [13], [14] have been applied to create a powerful model for predicting the price change volatility in Forex market. The developed models based on these algorithms yield approximately 20 to 50 percent a year. In a few years, Deep Learning, a subfield of Machine Learning research area has been introduced in the financial area [15], [16]. Considering the Forex historical data as a time-series data, an algorithm of Deep Learning that suit for this type of data is Recurrent Neural Networks (RNN), Long Short-Term Memory (LSTM) algorithm. In 2017, the novel two model-based LSTM are developed by Bao et al. [17] and Yang et al. [18]. The model of Bao et al. yields yearly average $45.4 \%$ for Nifty 50 Index, $59.4 \%$ for Nikkei 225 Index and $64.0 \%$ for DJIA Index, while the financial evaluation does not show for the model of Yang et al. Later, in 2018, Baek and Kim [19], Kim and Won [20] and Fischer and Krauss [20] also propose model-based LSTM algorithm. Their evaluation results based on stock historical data show that their model work-well in terms of the less error in prediction accuracy. However, only Fischer and Krauss show the results in term of financial evaluation. Another research that applies Deep learning algorithm in the financial area is conducted by Sezer and Ozbayoglu [21] in 2018. Their idea is to convert time-series data to be an image and exploit the Convolution Neural Network (CNN) algorithm to analyze the small pattern in the image. Their experimental results in term of financial aspect show that they yield approximately $12.59 \%$ annually for Dow 30 and $13.01 \%$ for ETFs. As we focus on the Forex market and 
based on the information described above, there is a lag of research that applied AI technology particularly Deep Learning algorithm in the Forex market. Therefore, in this paper, we propose a novel model based on CNN algorithm that aims to accurately predict the daily movement of the Forex price.

The rest of this paper is organized as follows. Section II describes the data and the methodology used for conducting this research. The empirical results are discussed in section III. Finally, section IV represents our conclusions and makes suggestions for future research.

\section{MATERIALS AND METHODS}

\section{A. Research Framework}

In this paper, we propose a Deep Learning model based on an image processing approach for predicting the daily price movement of the Forex market. The accuracy of the prediction is compared with the accuracy of the model-based Machine Learning algorithms including C5.0 [22], Logistic Regression [23], Discriminant [24], ANN [25], CHAID [26] and C\&R Tree [27]. For Deep Learning model, the experimental process is divided into six steps including data collection, data preparation, data transformation, data labeling, modeling, performance evaluation and comparison, and financial evaluation. For Machine Learning model, the experimental process is divided into four steps including data collection, data preparation, and data labeling, modeling, and performance evaluation and comparison. The framework that we use for conducting this research is illustrated in Fig. 1.

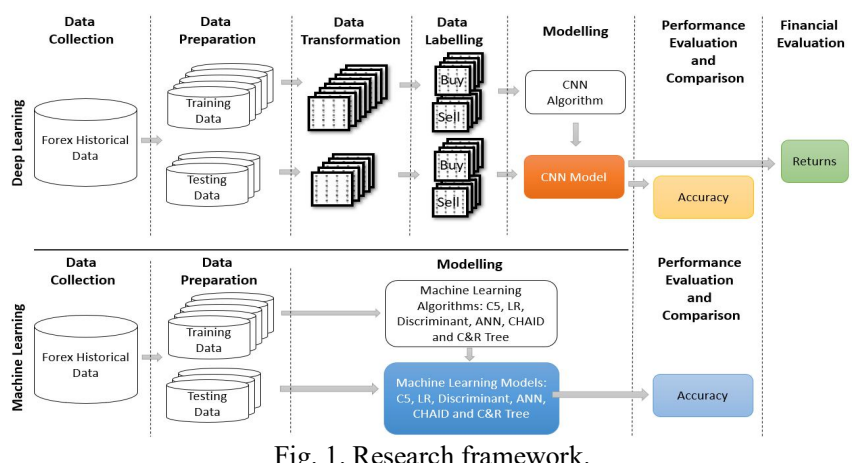

Fig. 1. Research framework.

\section{B. Data Collection}

The dataset uses for conducting this research is Forex historical data of the EURUSD currency pairwise from 3 January 2000 to 14 September 2018. The data is exported from a Forex trading platform called Meta Trader 4 (MT4) of FXCM [28] using time-frame daily. Therefore, the dataset consists of approximately 4,865 records.

\section{Data Preparation}

The dataset is divided into training and testing set using sliding-windows technique [29] as shown in Fig. 1. This technique is used for preventing the model from the model overfitting issue. We use the year 2000 to 2004 as a training dataset and use the year 2005 as a testing dataset. Then, we move forward for 1 year. In addition, we create another dataset, which consists of the training set $(2000-2016)$ and testing set $(2017-2018)$. As a result, we obtain totally 14 datasets. All 14 dataset consists of approximately 25,300 records.

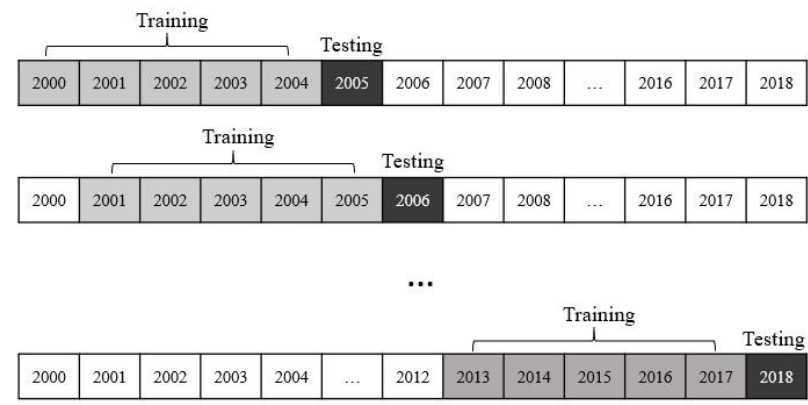

Fig. 2. Dividing data into training and testing set using the sliding-windows technique.

\section{Data Transformation}

In this process, the data are converted to be $16 \times 16$ pixels grayscale images. An image represents a daily data of the dataset. In order to create an image, which contains $16 \times 16$ pixels, we use 15 technical indicators and chart information. For each indicator, 16 different input of parameter is used in order to obtain 16 different values of each indicator. Fig. 3 describe the structure of daily data that is used to create a $16 \times 16$ pixels image.

TABLE I: LIST OF 15 TECHNICAL INDICATORS

\begin{tabular}{ll}
\hline \hline Category & Indicator \\
& Relative Strength Index (RSI) \\
& Stochastic Oscillator (STO) \\
Momentum & Williams \%R (WR) \\
& Awesome Oscillator (AO) \\
& True strength index (TSI) \\
& Money Flow Index (MFI) \\
\hline \multirow{2}{*}{ Volatility } & Average True Range (ATR) \\
& Bollinger Bands (BB) \\
\hline \multirow{3}{*}{ Trend } & Moving Average Convergence Divergence (MACD) \\
& Average Directional Movement Index Indicator (ADX) \\
& Commodity Channel Index (CCI) \\
& $\begin{array}{l}\text { Detrended Price Oscillator (DPO) } \\
\text { Exponential Moving Average (EMA) }\end{array}$ \\
\hline Volume & Force Index (FI) \\
\hline
\end{tabular}

16 different parameters

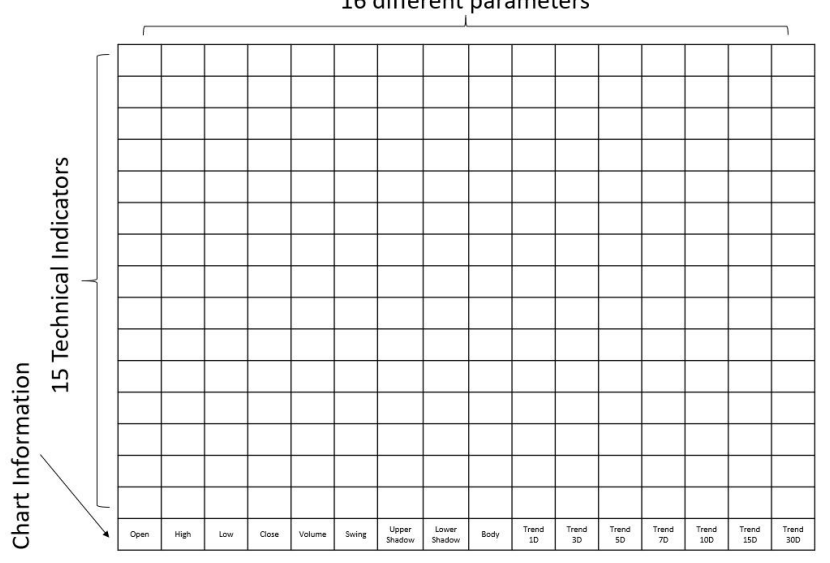

Fig. 3. Dividing data into training and testing set using the sliding-windows technique.

The 15 technical indicators are selected from the four 
different categories including Momentum, Volatility, Trend, and Volume. Table I show a list of all indicators grouped by its category.

For the 16th row, which is the chart information (in Fig. 3) consist of 16 columns. The first 9 columns are the information we create from a candlestick and daily trading volume as shown in Fig. 4, otherwise are trends. We create trend values of $1,3,5,7,10,15$, and 30 days. Value 1 refers to uptrend, and value -1 refers to downtrend as illustrated in Fig. 5.

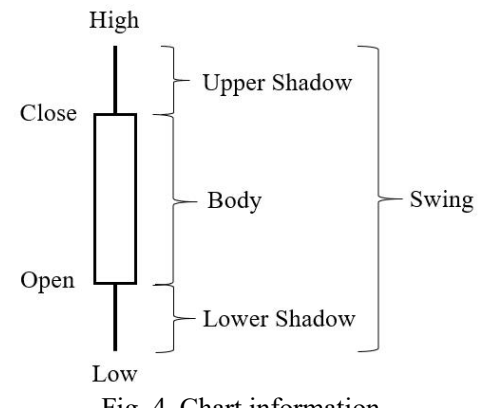

Fig. 4. Chart information.

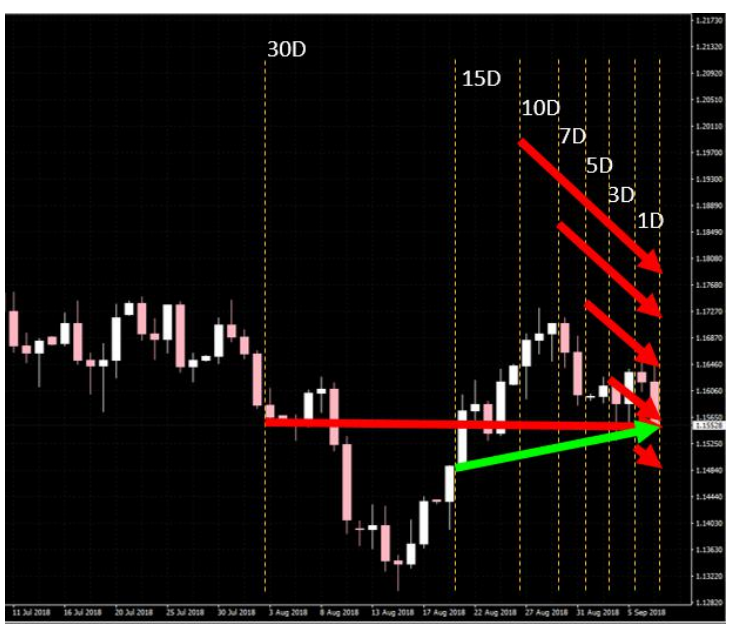

Fig. 5. Example of how we create trend values.

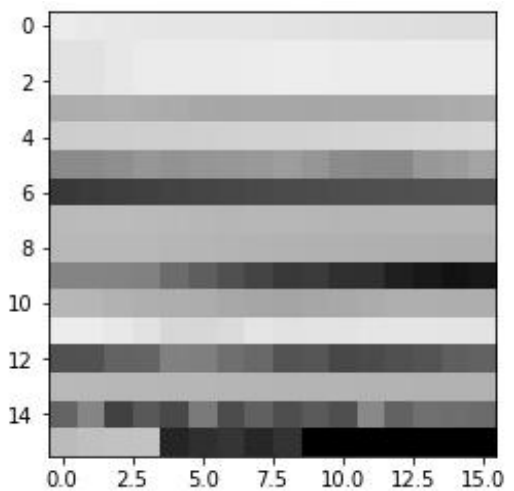

Fig. 6. Dividing data into training and testing set using the sliding-windows technique.

In order to create a grayscale image, Min-Max Normalization technique [30] is employed to scale the data between $0-255$. Min-max normalization is often known as feature scaling where the values of a numeric range of a feature of data are increased or reduced to a scale between the new maximum and the new minimum. Therefore, the new value of the member of the set of observed values is calculated according to the new maximum and the new minimum by using the following equation: $v_{\text {iew }}=(v-\min /(\max -\min ))\left(\max _{\text {new }}-\min _{\text {new }}\right)+\min _{\text {new }}$

where $v$ is a member of the set of observed values, min and max are the minimum and maximum values in the range of the set of observed values, $\max _{\text {new }}$ and $\min _{\text {new }}$ are the minimum and maximum values in the range of the new values.

Then the image data are created using the PIL library in Python [31]. At the end of the data transformation process, we obtain the $16 \times 16$ pixels grayscale images as shown in Fig. 6.

\section{E. Data Labeling}

The majority goal of this research is to predict the daily movement of Forex, which is "Up" and "Down". Up means, the price is closed above the open price, and vice versa for Down. Suppose that if we know that tomorrow price will be moved Up, we will send a Buy ticket today, and tomorrow we got a profit. For this reason, we create a signal column, which is the target class that we attempt to make a prediction. Firstly, the values of the signal column are created using the following formula:

$$
\text { Signal }=\left\{\begin{array}{c}
\text { Buy, if close }- \text { open }>0 \\
\text { Sell, if close }- \text { open }<0
\end{array}\right.
$$

Then, we lagging-time for 1 day of the signal column. This means that we use today information with tomorrow label according to the signal class. As a result, we can label whether an image is Buy or Sell as illustrated in Fig. 7.

\section{F. Modeling}

After we transform data into images, we use the training set of image data to build the prediction model. The model is based on Convolution Neural Network [32] (Fig. 8), which is one of the Deep Learning techniques for analyzing an image. In this section, the details of the proposed CNN model are described.

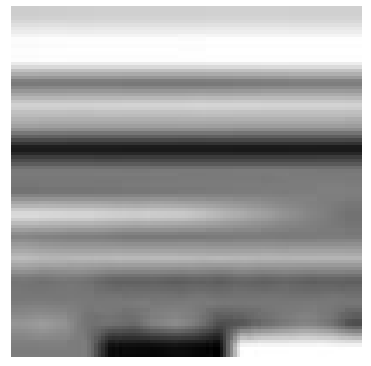

Buy

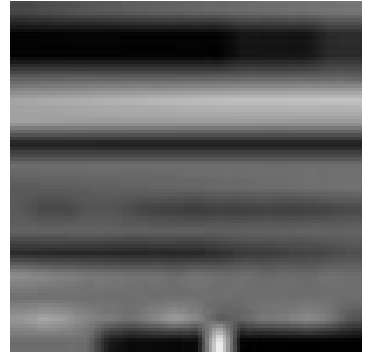

Sell
Fig. 7. Example of images of the buy and sell signals.

The proposed $\mathrm{CNN}$ contains nine layers including an input layer $(16 \times 16)$, three convolutional layers $(16 \times 16 \times 16,16$ $\times 16 \times 32$, and $16 \times 16 \times 64)$, two max pooling $(7 \times 7 \times 64$, $7 \times 7 \times 64)$, a dropout layer (0.25), a fully connected layer (128) and an output layer (2). The filter size is $3 \times 3$. A Dropout layer is added to prevent overfitting [33]. In this research, the proposed CNN model is implemented using Keras, Tensorflow infrastructure in the Python language. 
Using 500 epochs to train the model.

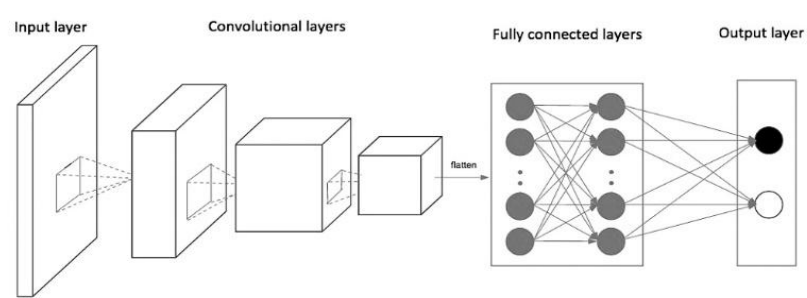

Fig. 8. Structure of the general convolution neural network.

\section{G. Performance Evaluation}

The performance of the proposed CNN model is evaluated in term of accuracy of the prediction. The accuracy can be computed using the following equation:

$$
\text { Accuracy }=(T P+T N) /(T P+F N+F P+T N)
$$

where $T P$ is the number of true positives, $T N$ is the number of true negatives, $F P$ is the number of false positives, and $F N$ is the number of false negatives. $T P, T N, F P$, and $F N$ are derived from the Confusion Matrix table, which is the result of performance evaluation of the models. The example of the Confusion Matrix table is shown in Table II.

\begin{tabular}{|} 
TABLE II: CONFUSION MATRIX TABLE \\
\begin{tabular}{|c|c|c|c|}
\hline \multicolumn{2}{|c|}{} & \multicolumn{2}{c|}{ Actual Data } \\
\cline { 3 - 4 } \multicolumn{2}{|c|}{} & Positive & Negative \\
\hline \multirow{2}{*}{ Predicted Data } & Positive & TP & FP \\
\cline { 2 - 4 } & Negative & FN & TN \\
\hline
\end{tabular}
\end{tabular}

The accuracy of the proposed model is compared to other machine learning techniques including C5.0, Artificial Neural Network, Logistic Regression, and Discriminant.

\section{H. Financial Evaluation}

In order to evaluate the performance of the model in term of the financial aspect, the predicted Buy and Sell are made. Then, the profits or loses of each transaction is calculated by the following formula:

$$
\text { Profit }_{i}=\mid \text { Close }- \text { Open }- \text { Spread } \mid \times \text { Lot_size }
$$

Note that if the predicted signal is false, the Profit ${ }_{i}$ is considered to be a negative return. Therefore, the Profit ${ }_{i}$ that calculates according to the equation (4) has to be multiplied by -1 . In addition, we set $\$ 1,000$ USD an initial balance, and the trading lot size is equal to 0.1 of ECN account.

At the end of the calculating process, the total balance is calculated by summarizing the initial balance and the profit of each transaction as illustrated in equation 5 .

$$
\text { TotalBlance }=\text { Initialblance }+\sum_{i=1}^{n} \text { Profit }_{i}
$$

\section{RESULTS AND DISCUSSIONS}

\section{A. Performance Evaluation}

Table III shows the results of performance evaluation in term of accuracy. As is illustrated in Table III, the accuracy of all testing scenarios seems to be consistent. The 14 th scenario provides the highest accuracy at approximately $77.48 \%$, while the lowest accuracy is 70.71 when we use the year $2009-2013$ as the training data and use the year 2014 as the testing data.

TABLE III: ACCURACY OF THE PREDICTIONS

\begin{tabular}{cccc}
\hline \hline Scenario & Training Data & Testing Data & Accuracy (\%) \\
\hline 1 & $2000-2004$ & 2005 & 73.68 \\
2 & $2001-2005$ & 2006 & 72.73 \\
3 & $2002-2006$ & 2007 & 70.40 \\
4 & $2003-2007$ & 2008 & 73.89 \\
5 & $2004-2008$ & 2009 & 75.45 \\
6 & $2005-2009$ & 2010 & 75.74 \\
7 & $2006-2010$ & 2011 & 73.71 \\
8 & $2007-2011$ & 2012 & 74.34 \\
9 & $2008-2012$ & 2013 & 72.60 \\
10 & $2009-2013$ & 2014 & 70.71 \\
11 & $2010-2014$ & 2015 & 73.51 \\
12 & $2011-2015$ & 2016 & 74.11 \\
13 & $2012-2016$ & 2017 & 76.87 \\
14 & $2000-2016$ & $2017-2018$ & 77.48 \\
\hline \hline
\end{tabular}

Fig. 9 shows the results in term of confusion matrix of the model that is created using data from 2000 to 2016 and testing data from 2017 to 2018 . As can be seen in Fig. 9, for Buy signals, the model accurately predicts 1,054 times (39.8\%), while it fails 258 times (9.7\%). For Sell signal, the model accurately predicts 997 times $(37.7 \%)$, while it fails 338 times (12.8\%). Overall the model accurately predicts $77.5 \%$ and fails $22.5 \%$.

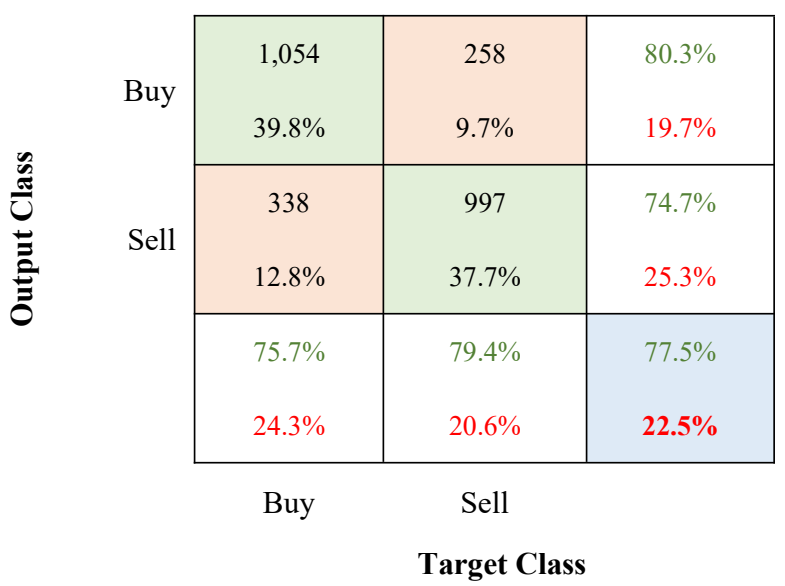

Fig. 9. Confusion Matrix of the $14^{\text {th }}$ scenario.

\section{B. Performance Comparison}

The accuracy of the proposed CNN model is compared with other Machine Learning algorithms as demonstrated in Fig. 10.

As can be seen in Fig. 10, the model that provides the highest accuracy is C5.0 (77.67\%) followed by our proposed CNN model (77.48\%), Logistic Regression model (75.93\%) and discriminant model $(75.93 \%)$, respectively. Based on this result, it could be concluded that the proposed model performs well compared to other Machine Learning models. 
However, C5.0 provide a better performance than the proposed $\mathrm{CNN}$ model. This result from the possibility that using a rule-based algorithm for creating a model for predicting the time-series data could provide a better result than using the image processing-based approach. However, the result is extremely trivial.

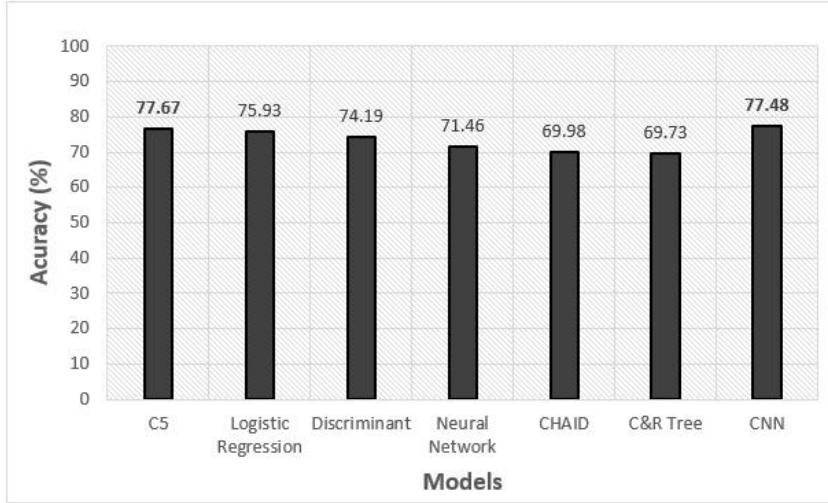

Fig. 10. The accuracy of the CNN model compared with machine learning techniques.

\section{Financial Evaluation}

In this section, we show the performance of the proposed CNN model in term of the financial aspect. The best model in scenario 14th is used in this experiment. We set up the initial balance at \$1,000USD. Trading account is ECN account with leverage 1:400. The spread is 1 pip. Lot size is 0.1 . The result shows that from January 2017 to September 2018 (Approximately 442 days), the proposed CNN model can produce profits of approximately \$68,353USD as demonstrated in Fig. 11.

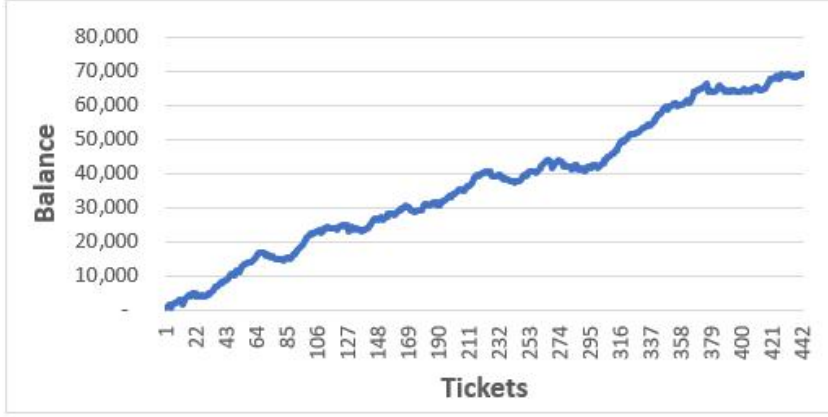

Fig. 11. Financial Accumulation of the CNN model.

\section{CONCLUSION}

In this paper, we proposed a novel model based on $\mathrm{CNN}$ that can be effectively used as a tool for predicting the daily movement of the Forex. We propose a technique for transforming the financial time series data into image data that can be used with $\mathrm{CNN}$ algorithm. The results of the performance evaluation in term of accuracy of the prediction shows that the proposed $\mathrm{CNN}$ model performs well compared to other model-based Machine Learning algorithms. Although C5.0 model performs better than the proposed CNN model, it is different in a trivial percentage. In addition, the result in term of financial evaluation also confirms that the proposed $\mathrm{CNN}$ model has a great potential to make profits for Forex traders.
For future works, we aim to enhance the performance of the model by tune-up the structure of the CNN model. In addition, we expect to implement the system that can be used in the real-world and can automatically provide a daily signal according to the predicting signal from the proposed model.

\section{CONFLICT OF INTEREST}

The authors declare no conflict of interest.

\section{AUTHOR CONTRIBUTIONS}

Watthana Pongsena conducted the research and wrote the paper. All authors had approved the final version of the paper.

\section{ACKNOWLEDGMENT}

The authors are grateful to National Research Council of Thailand, Ministry of Science and Technology, and Suranaree University of Technology for granting the research funds.

\section{REFERENCES}

[1] L. L. Jacque, "Management and control of foreign exchange risk," Springer Science \& Business Media, 2013.

[2] J. S. Abarbanell and B. J. Bushee, "Fundamental analysis, future earnings, and stock prices," J. Account. Res., vol. 35, no. 1, pp. 1-24, 1997.

[3] J. J. Murphy, Study Guide for Technical Analysis of the Futures Markets: A Self-training Manual, New York Institute of Finance New York, 1987.

[4] A. W. Lo, H. Mamaysky, and J. Wang, "Foundations of technical analysis: Computational algorithms, statistical inference, and empirical implementation," J. Finance, vol. 55, no. 4, pp. 1705-1765, 2000.

[5] B. W. Silverman, "Density estimation for statistics and data analysis," Routledge, 2018.

[6] T. Young, Moving Average Convergence Divergence (MACD).

[7] T. T.-L. Chong and W.-K. Ng, "Technical analysis and the London stock exchange: Testing the MACD and RSI rules using the FT30," Appl. Econ. Lett., vol. 15, no. 14, pp. 1111-1114, 2008.

[8] R. Rosillo, D. la Fuente, and J. A. L. Brugos, "Technical analysis and the Spanish stock exchange: testing the RSI, MACD, momentum and stochastic rules using Spanish market companies," Appl. Econ., vol. 45, no. 12, pp. 1541-1550, 2013.

[9] E. Guresen, G. Kayakutlu, and T. U. Daim, "Using artificial neural network models in stock market index prediction," Expert Syst. Appl., vol. 38, no. 8, pp. 10389-10397, 2011

[10] R. J. Kuo, C. H. Chen, and Y. C. Hwang, "An intelligent stock trading decision support system through integration of genetic algorithm based fuzzy neural network and artificial neural network," Fuzzy sets Syst., vol. 118, no. 1, pp. 21-45, 2001.

[11] J. L. Ticknor, "A Bayesian regularized artificial neural network for stock market forecasting," Expert Syst. Appl., vol. 40, no. 14, pp. 5501-5506, 2013.

[12] L. Kryzanowski, M. Galler, and D. W. Wright, "Using artificial neural networks to pick stocks," Financ. Anal. J., pp. 21-27, 1993.

[13] K. Kim and I. Han, "Genetic algorithms approach to feature discretization in artificial neural networks for the prediction of stock price index," Expert Syst. Appl., vol. 19, no. 2, pp. 125-132, 2000.

[14] F. Allen and R. Karjalainen, "Using genetic algorithms to find technical trading rules1," J. Financ. Econ., vol. 51, no. 2, pp. 245-271, 1999.

[15] S. Galeshchuk and S. Mukherjee, "Deep networks for predicting direction of change in foreign exchange rates," Intell. Syst. Accounting, Financ. Manag., vol. 24, no. 4, pp. 100-110, 2017.

[16] J. Korczak and M. Hemes, "Deep learning for financial time series forecasting in A-Trader system," in Proc. Federated Conference on Computer Science and Information Systems (FedCSIS), 2017, pp. 905-912.

[17] W. Bao, J. Yue, and Y. Rao, “A deep learning framework for financial time series using stacked autoencoders and long-short term memory," PLoS One, vol. 12, no. 7, p. e0180944, 2017. 
[18] H. Yang, Z. Pan, and Q. Tao, "Robust and adaptive online time series prediction with long short-term memory," Comput. Intell. Neurosci., 2017.

[19] Y. Baek and H. Y. Kim, "ModAugNet: A new forecasting framework for stock market index value with an overfitting prevention LSTM module and a prediction LSTM module," Expert Syst. Appl., vol. 113, pp. $457-480,2018$.

[20] H. Y. Kim and C. H. Won, "Forecasting the volatility of stock price index: A hybrid model integrating LSTM with multiple GARCH-type models," Expert Syst. Appl., vol. 103, pp. 25-37, 2018.

[21] T. Fischer and C. Krauss, "Deep learning with long short-term memory networks for financial market predictions," Eur. J. Oper. Res., vol. 270, no. 2, pp. 654-669, 2018.

[22] O. B. Sezer and A. M. Ozbayoglu, "Algorithmic financial trading with deep convolutional neural networks: Time series to image conversion approach," Appl. Soft Comput., 2018.

[23] T. Bujlow, T. Riaz, and J. M. Pedersen, "A method for classification of network traffic based on C5. 0 Machine Learning Algorithm," in Proc. International Conference on Computing, Networking and Communications, 2012, pp. 237-241.

[24] S. Dreiseitl and L. Ohno-Machado, "Logistic regression and artificial neural network classification models: a methodology review," $J$. Biomed. Inform., vol. 35, no. 5-6, pp. 352-359, 2002.

[25] E. Alpaydin, Introduction to Machine Learning, MIT press, 2009.

[26] Y. Kara, M. A. Boyacioglu, and Ö. K. Baykan, "Predicting direction of stock price index movement using artificial neural networks and support vector machines: The sample of the Istanbul Stock Exchange," Expert Syst. Appl., vol. 38, no. 5, pp. 5311-5319, 2011.

[27] M. Z. Noghabi, M. Ashoori, and M. Farshad, "Predicting the price of tehran stock market using data mining algorithms," Majlesi J. Mechatron. Syst., vol. 4, no. 4, 2015.

[28] C. Ferri, P. Flach, and J. Hernández-Orallo, "Learning decision trees using the area under the ROC curve," in Proc. ICML, 2002, vol. 2, pp. 139-146.

[29] E. Keogh, S. Chu, D. Hart, and M. Pazzani, "An online algorithm for segmenting time series," in Proc. EEE International Conference on Data Mining, 2001, pp. 289-296.

[30] L. Al Shalabi, Z. Shaaban, and B. Kasasbeh, "Data mining: A preprocessing engine," J. Comput. Sci., vol. 2, no. 9, pp. 735-739, 2006.

[31] M. F. Sanner and others, "Python: A programming language for software integration and development," J Mol Graph Model, vol. 17, no. 1, pp. 57-61, 1999.

[32] A. Krizhevsky, I. Sutskever, and G. E. Hinton, "Imagenet classification with deep convolutional neural networks," in Advances in Neural Information Processing Systems, 2012, pp. 1097-1105.

[33] N. Srivastava, G. Hinton, A. Krizhevsky, I. Sutskever, and R. Salakhutdinov, "Dropout: A simple way to prevent neural networks from overfitting," J. Mach. Learn. Res., vol. 15, no. 1, pp. 1929-1958, 2014.
Copyright (C) 2020 by the authors. This is an open access article distributed under the Creative Commons Attribution License which permits unrestricted use, distribution, and reproduction in any medium, provided the original work is properly cited (CC BY 4.0).

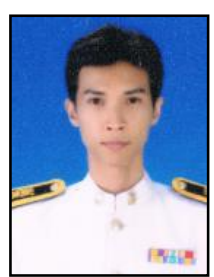

Watthana Pongsena is a Ph.D. student in the School of Computer Engineering, Suranaree University of Technology (SUT), Thailand. He received his B.E. and M.E. in computer engineering from Suranaree University of Technology, Thailand, in 2008 and 2012. His research of interest includes software engineering, data mining, artificial intelligence, and human-computer interaction.

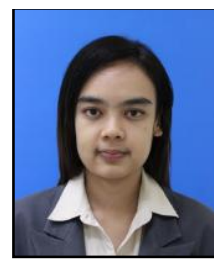

Prakaidoy Sitsayabut is a Ph.D. student in the School of Biotechnology, Institute of Agricultural Technology, Suranaree University of Technology, Thailand. She graduated with a bachelor degree of animal production of technology from the Suranaree University of Technology in 2006. After graduation, in 2012, she graduated with a master degree from the School of Biotechnology, Institute of Agricultural Technology, Suranaree University of Technology (SUT). Currently, her research of interest includes animal reproductive technology and data mining.

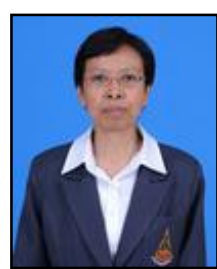

Nittaya Kerdprasop is an associate professor and the head of Data Engineering Research Unit, School of Computer Engineering, Suranaree University of Technology (SUT), Thailand. She received her B.S. in radiation techniques from Mahidol University, Thailand, in 1985, M.S. in computer science from the Prince of Songkla University, Thailand, in 1991 and Ph.D. in computer science from Nova Southeastern University, U.S.A., in 1999. Her research of interest includes data mining, artificial intelligence, logic, and constraint programming.

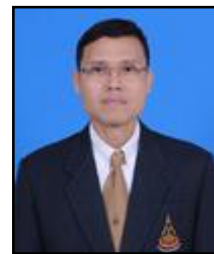

Kittisak Kerdprasop is an associate professor at the School of Computer Engineering, chair of the School, and the head of Knowledge Engineering Research Unit, SUT. He received his bachelor degree in mathematics from Srinakarinwirot University, Thailand, in 1986, MS in computer science from the Prince of Songkla University, Thailand, in 1991 and Ph.D. in computer science from Nova Southeastern University, U.S.A., in 1999. His current research includes machine learning and artificial intelligence. 